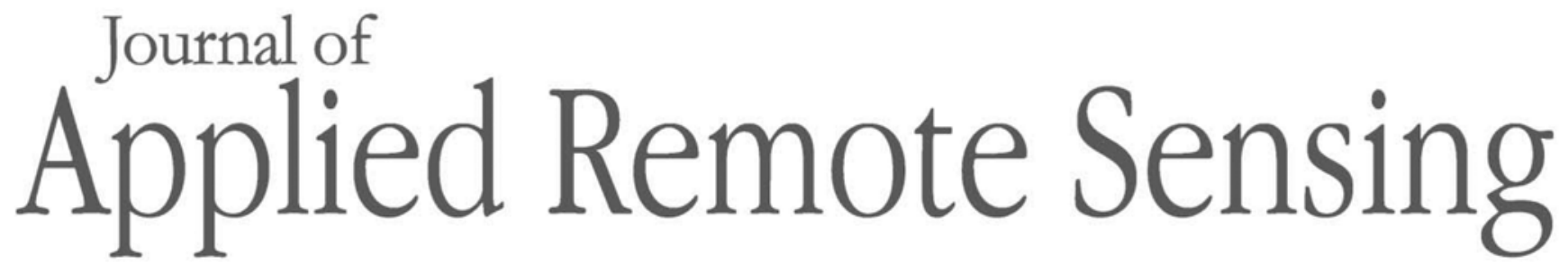

RemoteSensing.SPIEDigitalLibrary.org

\title{
Scalability conjecture for the Fried parameter in synthesis of turbulent atmosphere point spread functions
}

Bobby R. Hunt
Amber L. Iler
Michael A. Rucci 


\title{
Scalability conjecture for the Fried parameter in synthesis of turbulent atmosphere point spread functions
}

\author{
Bobby R. Hunt, ${ }^{a, *}$ Amber L. Iler, ${ }^{b, *}$ and Michael A. Rucci ${ }^{\mathrm{c}}$ \\ ${ }^{a}$ Integrity Applications Incorporated-Pacific Defense Solutions, Kihei, Hawaii, United States \\ ${ }^{b}$ Integrity Applications Incorporated, Ann Arbor, Michigan, United States \\ ${ }^{c}$ Air Force Research Laboratory, Wright Patterson Air Force Base, Ohio, United States
}

\begin{abstract}
A common method for synthesizing turbulent imagery is to model phase perturbations on a wavefront and then propagate the wavefront to the entrance pupil of an imaging system. The point spread function (PSF) that results from the wavefront in the pupil is then computed and used to synthesize images by the usual means of convolution. In a recent publication, a method was disclosed using sparse and redundant dictionaries of turbulent characteristics to construct PSFs directly in the image plane and simulate image formation without making phase models and computing wavefront propagation. However, the dictionary method, as disclosed in the recent publication, is limited to modeling PSFs characterized by the Fried parameter of the data used to construct the dictionary. Herein, we demonstrate that a dictionary constructed from data with a given Fried parameter can be scaled to construct turbulent PSFs corresponding to larger and smaller values of the Fried parameter. This enables a single dictionary, or a small number of dictionaries, to serve for the simulation of turbulent images over a range of turbulence conditions. () The Authors. Published by SPIE under a Creative Commons Attribution 3.0 Unported License. Distribution or reproduction of this work in whole or in part requires full attribution of the original publication, including its DOI. [DOI: 10.1117/1.JRS.12.042402]
\end{abstract}

Keywords: image modeling and simulation; turbulence; sparse and redundant representations; point spread functions; atmospheric optics; remote sensing; image restoration.

Paper 180015SS received Jan. 5, 2018; accepted for publication Mar. 28, 2018; published online Jul. 5, 2018.

\section{Introduction and Motivation of Research}

\subsection{Collecting Imagery through Atmospheric Turbulence}

Random fluctuations in temperature, pressure, and turbulent movement lead to turbulent effects of warping and blurring in imagery through random fluctuations in the index of refraction. ${ }^{1}$ The refractive index structure function, $C_{n}^{2}$, conveys information on the strength of the turbulence. In addition, $C_{n}^{2}$ can be a scalar value in horizontal imaging or a vertical profile, in the astronomy case, which can be used to calculate the isoplanatic angle and the Fried parameter. These index of refraction fluctuations occur rapidly, and a common manner to conceptualize these effects is by the atmosphere imposing an instantaneous point spread function (PSF) upon the entire image formation process that creates a point source image in the focal plane of an optical system. This instantaneous PSF implies a focal plane exposure that is brief enough to "freeze" the atmospheric turbulent effects. Describing, modeling, and synthesizing such PSFs are the objectives of this paper.

In astronomical imaging, the field of view of the camera, coupled with the greater intensity of turbulence near the camera, leads to an isoplanatic imaging case. Thus, a single, turbulent, space invariant, PSF exists that gives rise to warp and blur phase components across large parts of the image. In contrast, the horizontal imaging case has much more turbulence distributed between the camera and the object. In this configuration, the PSF is no longer spatially invariant and the

*Address all correspondence to: Bobby R. Hunt, E-mail: bhunt@integrity-apps.com; Amber L. Iler, E-mail: ailer@integrity-apps.com 
image has locally varying warp and blur, leading to an anisoplanatic imaging case. A third imaging scenario is where the camera is elevated, looking down at the ground, and a majority of the turbulent effects occur near the object. ${ }^{2,3}$ This case is also anisoplanatic in nature with spatially varying PSFs but to a greater degree than the horizontal imaging case.

The Fried parameter, $r_{0}$, is a measure that dictates the coherence diameter of the atmosphere. A larger Fried parameter corresponds to a smaller distortion being imposed on a focal plane image by turbulent atmosphere, and a weaker path integrated $C_{n}^{2}$ value. Furthermore, $r_{0}$ can be used to calculate the long and short exposure optical transfer functions (OTFs). These statistical representations of turbulence effects are well established in modeling. ${ }^{4}$

The short exposure can contain higher spatial frequency content, i.e., instantaneous amplitude and phase information, and is used in mitigation techniques such as the bispectral speckle technique ${ }^{5}$ or the block-matching Wiener filter ${ }^{6}$ to help estimate the spatially variant PSF used in the deconvolution step. These mitigation techniques, along with others, such as phase diversity and "lucky-look" imaging, all attempt to remove the effects of turbulence in imagery, which leads to stabilized imagery with improved resolution to see smaller details in imagery.

\subsection{Recent Developments in Signal Modeling and Representation}

Over the past 20 years, researchers have actively looked for alternatives to space invariant Fourier representations to support efficient modeling and signal processing. Developments during this era began with the use of alternative basis functions via wavelet transforms ${ }^{7}$ and progressed through recent compressed sensing developments for sparse representation of a signal without the constraints of the Shannon/Nyquist sampling theorem. ${ }^{8}$ While sparse representations have proven to be efficient at representing signals, uncertainty in or corruption of the transform coefficients can lead to serious misrepresentation of the signal. ${ }^{9}$ By including redundancy in the representation, additional robustness is added that can theoretically make the representation more accurate, as well as efficient. ${ }^{10}$ While the redundant counterpart to basis pursuit has existed since $1952,{ }^{9}$ efforts to combine sparse and redundant representations have led to new methods for representation of signals.

The advent of sparse and redundant representations for signal processing has led to questions of whether bases can be constructed directly from available data and whether such an approach might be capable of an even more accurate representation of the data. Aharon et al. compared image reconstructions from a sparse and redundant dictionary, trained on image data, to an overcomplete, separable, Haar dictionary and an overcomplete, discrete cosine transform (DCT) dictionary. ${ }^{11}$ Their findings showed that reconstructions of images corrupted by missing pixels were restored most accurately (with lowest root mean squared errors) when the data-derived dictionary was used, compared to the Haar and DCT dictionaries. ${ }^{11}$ In addition to restoration accuracy, they also looked at the efficiency of the three dictionaries for compressing the imagery to fewer bits per pixel versus the peak signal-to-noise ratio (PSNR). The data-derived dictionary achieved significantly higher PSNR (1 to $2 \mathrm{~dB}$ better) than the other two dictionaries for bit rates less than 1.5 bits per pixel. ${ }^{11}$ Since that time, others have further applied data-derived dictionary approaches for image denoising, demosaicking, and deblurring. ${ }^{12,13}$ The continued success of these approaches has demonstrated the potential of sparse and redundant dictionaries for image processing applications.

\subsection{Synthesis of PSFs from Dictionary Representation}

In our previous paper, ${ }^{3}$ we adapted the K-means singular value decomposition (K-SVD) algorithm used by Aharon et al. ${ }^{11}$ to derive a dictionary of PSF atoms from imagery of point sources impacted by deep turbulence. K-SVD is capable of achieving the best representation for each member of the training set with vectors that are as sparse as possible. Using 100 image frames, we trained a dictionary of $256 \mathrm{PSF}$ atoms, then we used the 256-atom dictionary to represent all the PSFs in the training set by means of the Orthogonal Matching Pursuit algorithm. ${ }^{14}$ The 256atom dictionary was so efficient at capturing PSF structure that a representation using only 64 coefficients was found to be accurate in representing an arbitrary PSF. The caveat to this level of sparsity was that not all 256 coefficient atoms were used with equal frequency. This led us to 
Hunt, ller, and Rucci: Scalability conjecture for the Fried parameter in synthesis of turbulent...

gather mean and standard deviation statistics for each of the dictionary atoms based on the training PSFs, which provided a statistical basis for weighting the coefficients when synthesizing new PSFs.

We synthesized new PSFs by generating new coefficients that maintained the same coefficient statistics as the original training set. New PSFs synthesized by this method were then compared to real PSFs that were collected under the same turbulence conditions as the training PSFs but not used in compiling the dictionary or deriving the weights. Visual comparison confirmed the appearance of the synthesized and real PSFs were consistent and additional statistical tests showed that PSFs synthesized using our dictionary process were representative of the same turbulence conditions used to construct the dictionary. Thus, through the use of only 64 coefficients in generating our synthesized PSFs, and by verifying the statistical similarities they had to real PSFs, our approach demonstrated the efficiency and accuracy of sparse and redundant representations for characterizing PSFs in the focal plane of the sensor. The shortfall of this approach is that the dictionary derived from a set of PSFs under a fixed value of the Fried parameter, $r_{0}$, will naturally synthesize PSFs with the same $r_{0}$ value.

\subsection{Objectives}

In this paper, we investigate the ability to achieve fast PSF synthesis without collecting new PSFs with different $r_{0}$ values. Such a capability would be helpful in cases where the exact $r_{0}$ for the turbulent imagery is not known and would also be beneficial to avoid collecting data and recompiling dictionaries for many different turbulence conditions. As a result, we also address the goal of performing dictionary PSF synthesis from physical-based parameters (in this case, the Fried parameter) that are used for statistical validation in other simulation techniques. ${ }^{4}$

The rest of the paper is organized as follows. Section 2 discusses the characteristics in Fried's parameter related to varying turbulence strength. The scaling of turbulent PSFs to match new values of Fried's parameter is discussed in Sec. 3. Section 4 contains the results and validation of the PSF scaling method we propose. Finally, the conclusion and suggestions for further effort can be found in Sec. 5 .

\section{Characteristics of PSFs from Different Turbulence Conditions}

\subsection{Collection of Turbulent PSF Datasets}

In September 2015, the EO Target Detection and Surveillance Branch of the Air Force Research Laboratory conducted a series of isoplanatic angle experiments at the Wright Patterson Air Force Base in Dayton, Ohio. The complete experimental details and collection mechanisms for those experiments, referred to as the "ISO" experiments, are presented in a companion article to this paper. $^{3}$ To summarize briefly, two point sources were created by a laser diode on the ground and were imaged through a nearly horizontal path by a camera in a tower $5.09 \mathrm{~km}$ distant. There was substantial turbulence upwelling directly from the ground underneath the path of the laser source to the collection system. This created large datasets with many observations of point sources in the focal plane of a fast-framing scientific camera, producing PSFs caused by the turbulent conditions prevailing during collection.

A total of 14 ISO collections were made during September 21 to 22, 2015. The pulse rate of the laser diodes and the frame rate of the camera were set to capture individual laser pulses within each data collect. One test dataset was collected on September 21, and 13 additional collections were made on September 22, 2015. The 13 PSF data collections from September 22 were labeled as Test 01, Test 02 , Test $03, \ldots$, up to Test 13 , as shown in Table 1 . Each collection contained 3000 frames of imagery of the point sources. The collection on September 21 took place at 13:53 local time. The collections on September 22 were spaced from midmorning (09:45) to later in the afternoon (17:03), leading to variations in the degree of turbulence that peaked in the middle of the day and decreased in the late afternoon. A scintillometer was placed adjacent to the test environment and was used to measure data for the computation of $C_{n}^{2}$ and the Fried parameter, $r_{0}$, for all the data collections. Derived measurements of $r_{0}$ averaged over each test indicated the 13 
Hunt, Iler, and Rucci: Scalability conjecture for the Fried parameter in synthesis of turbulent...

Table 1 Setup details for the 14 ISO data collections.

\begin{tabular}{|c|c|c|c|c|c|c|}
\hline ISO_TEST\# & Date, time & Aperture (in.) & Aperture (m) & $C_{n}^{2}\left(\mathrm{~m}^{-2 / 3}\right)$ & Measured $r_{0}(\mathrm{~m})$ & Derived $r_{0}(\mathrm{~m})$ \\
\hline Test01 (9/21) & $9 / 21,15: 53$ & 2.25 & 0.0572 & $1.1 e-14$ & $\mathrm{~N} / \mathrm{A}$ & $\mathrm{N} / \mathrm{A}$ \\
\hline Test01 (9/22) & 9/22, 09:45 & 2.25 & 0.0572 & $0.6 e-14$ & 0.0303 & 0.030 \\
\hline Test02 & $9 / 22,10: 03$ & 1.50 & 0.0381 & $0.7 e-14$ & 0.0292 & 0.030 \\
\hline Test03 & $9 / 22,10: 53$ & 2.25 & 0.0572 & $1.0 e-14$ & 0.0249 & 0.022 \\
\hline Test04 & 9/22, 11:07 & 1.50 & 0.0381 & $1.1 \mathrm{e}-14$ & 0.0253 & 0.023 \\
\hline Test05 & $9 / 22,11: 54$ & 2.25 & 0.0572 & $1.1 \mathrm{e}-14$ & 0.0218 & 0.023 \\
\hline Test06 ${ }^{a}$ & $9 / 22,12: 09$ & 1.50 & 0.0381 & $1.1 \mathrm{e}-14$ & 0.0214 & 0.025 \\
\hline Test07 & $9 / 22,13: 27$ & 2.25 & 0.0572 & $1.4 \mathrm{e}-14$ & 0.0210 & 0.021 \\
\hline Test08 & $9 / 22,13: 41$ & 1.50 & 0.0381 & $1.2 \mathrm{e}-14$ & 0.0169 & 0.023 \\
\hline Test09 & $9 / 22,14: 39$ & 2.00 & 0.0508 & $1.3 e-14$ & 0.0186 & 0.025 \\
\hline Test10 ${ }^{\mathrm{a}}$ & $9 / 22,14: 53$ & 2.50 & 0.0635 & $1.3 e-14$ & 0.0200 & 0.020 \\
\hline Test11 & $9 / 22,15: 46$ & 2.75 & 0.0699 & $1.0 e-14$ & 0.0239 & 0.030 \\
\hline Test12 ${ }^{\mathrm{a}}$ & $9 / 22,16: 03$ & 3.00 & 0.0762 & $0.9 e-14$ & 0.0232 & 0.030 \\
\hline Test13 ${ }^{a}$ & $9 / 22,17: 03$ & 2.25 & 0.0572 & $0.55 e-14$ & 0.0354 & 0.040 \\
\hline
\end{tabular}

${ }^{\mathrm{a}}$ Cases to be examined in more detail in this paper.

datasets on September 22 showed PSFs with a range of $r_{0}$ values from 0.02 to $0.04 \mathrm{~m}$, which are grossly consistent with the instantaneous scintillometer measurements. The method of computing the derived $r_{0}$ values from the collected PSFs, described in detail in Sec. 3.1, relied on the direct comparison of computed OTFs and the theoretical value of the OTF as a function of the Fried parameter.

\subsection{Characterization of Turbulent Point Source Functions}

Our experiments utilized four different collections from the full set of 14 , selected on the basis of the value of the $r_{0}$ parameter measured in the experiments. These measured values were confirmed by comparing the actual calculated OTFs of the test data to the theoretical, long-exposure OTF of imaging through turbulence as a function of the $r_{0}$ parameter, the wavelength, $\lambda$, the focal length, $F$, and the spatial frequency, $\rho^{1}$

$$
P(\rho)=\exp \left[-3.44\left(\frac{\lambda F \rho}{r_{0}}\right)^{\frac{5}{3}}\right]
$$

To calculate the long-exposure OTF for the collected PSFs, we Fourier transformed the PSFs and computed the average of their complex Fourier values. The real component of the average significantly dominated the complex component, so the magnitude of the average was computed and retained. ${ }^{1}$ This magnitude was used to normalize all the Fourier values to unity at the origin, creating a quantity analogous to the average OTF corresponding to the collection of PSFs. We plotted these numerically computed values in comparison to theoretical OTF values, as computed by Eq. (1), in order to derive the experimental value of $r_{0}$ with respect to the theoretical value.

As an example, Fig. 1 shows one result of this analysis. The dotted red line is the theoretical OTF computed from Eq. (1) for $r_{0}=0.02 \mathrm{~m}$, the dashed green line is the theoretical OTF for $r_{0}=0.03 \mathrm{~m}$, the dash-dotted blue line is the theoretical OTF for $r_{0}=0.04 \mathrm{~m}$, and the solid 


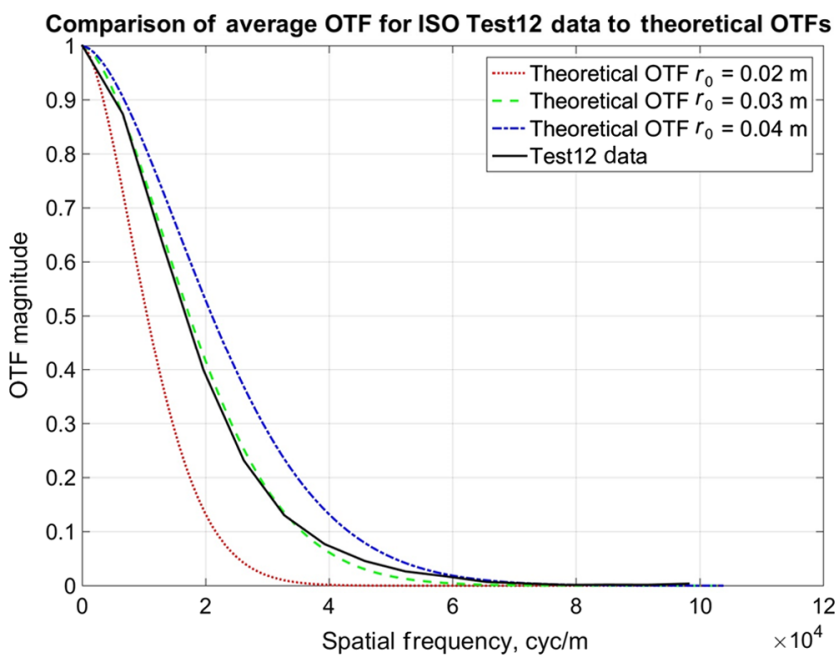

Fig. 1 By comparing theoretical (dashed lines) and experimentally computed (solid black line) turbulent OTFs, we are able to estimate the $r_{0}$ value of the experimental data.

black line is the experimental OTF calculated for Test12. Because the experimental OTF calculated from Test12 data lies so close to the theoretical OTF, we estimated the Test12 data OTF corresponded to $r_{0}=0.03 \mathrm{~m}$. Using similar comparisons for the other datasets, the experimental values of the $r_{0}$ parameter were quickly and easily estimated. We show these values for the 13 datasets collected on September 22 in the right-most column of Table 1.

Test06 and Test10 datasets were collected near the middle of the day (local solar noon was 13:29 on September 21 to 22) through early afternoon, when the solar influx over the optical path from emitter to receiver was at a maximum. Experiment personnel collected the Test 12 and Test13 datasets hours later in the afternoon, when the solar influx had significantly lessened. The decrease in solar flux from midday to afternoon results in decreased heating of the terrain under the optical path from laser sources to the tower receiver. This explains why the smallest values of $r_{0}$ occurred during Test10, with increasing values of $r_{0}$ being present in the experimental collections of the later afternoon. We note the derived, long-exposure values of $r_{0}$ were consistent with, but not equivalent to, the instantaneous measurements from the scintillometer, as recorded in Table 1. The differences were judged as being of the order of the experimental measurement errors in the $C_{n}^{2}$ values and were within the statistical variability of $r_{0}$ values as computed from $C_{n}^{2}$ values.

\subsection{Examples of PSFs from ISO Dataset Collections}

We began our analysis of PSFs by examining their structural characteristics under the differing turbulence conditions encountered during the ISO dataset collections. The values of $C_{n}^{2}$ for measurements on September 22 increase from midmorning (10:00) through $\sim 15: 00$, then decrease for the final three datasets, according to Table 1. In Fig. 2, we show several randomly selected instantaneous PSFs for the four test sets indicated by an "a" in column 1 of Table 1. Looking at these PSFs, we observe a similarity in variety of structural characteristics: some PSFs are more compact and others are more "splattered" or distributed in the focal plane due to the effects of turbulence. Also obvious is a tendency for difference in PSF sizes across the four test sets, which have been ordered from lowest $r_{0}$ value [Fig. 2(a)] to highest [Fig. 2(d)]. These variations in size and distribution of structure are typical of instantaneous PSFs. In general, the PSFs decrease in physical extent in pixels from top to bottom. Close examination of the PSFs from Test10 and Test06 compared to the Test13 PSFs suggests that the average size of the Test13 PSFs is distinctly smaller than the others. While similar shapes and structures are seen, the Test13 PSFs are, overall, more compact than either of the other datasets. This is consistent with the values of $C_{n}^{2}$ for these test sets, which indicate Test10 and Test06 experienced nearly twice the level of 
(a)

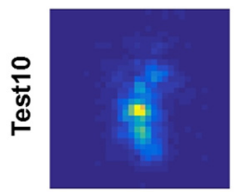

(b)

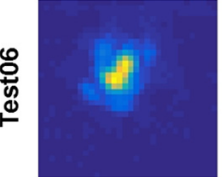

(c)

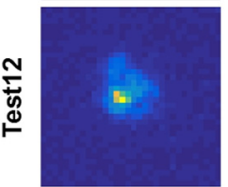

(d)

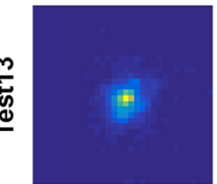


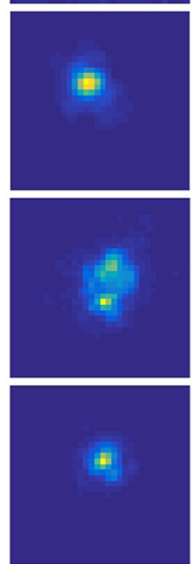
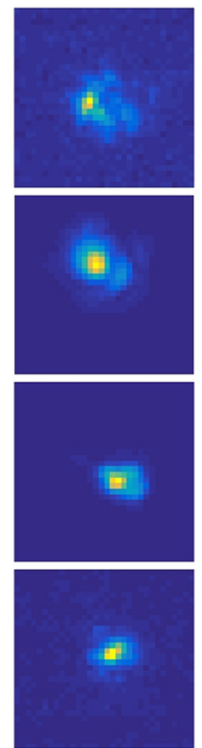
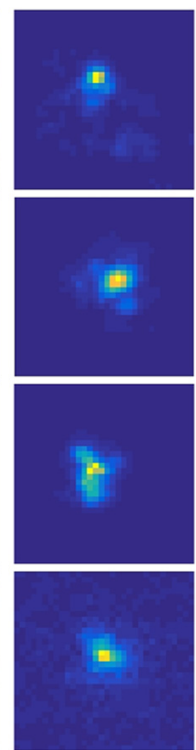

Fig. 2 A comparison of randomly selected point sources observed for selected ISO collections, ordered by increasing $r_{0}$ value: (a) Test10, (b) Test06, (c) Test12, and (d) Test13.

turbulence of Test13. Similar correlations in PSF size and turbulence conditions have been observed in previous, long-exposure, anisoplanatic measurements over a horizontal path. ${ }^{15-17}$

\subsection{Scalability Conjecture}

We now consider the physically meaningful interpretation of the Fried parameter: $r_{0}$ is the dimension of a clear (no turbulence) aperture that has the same resolution as the long-time-average image through an aperture, $D$, with turbulence present. From this, we expect $r_{0}<D$, and that, as the strength of turbulence increases, the Airy disk of a clear aperture diameter will become increasingly submerged by the "speckling" of turbulence. This implies that as the strength of turbulence increases, $r_{0}$ will decrease, and we should expect a commensurate increase in the physical dimension of the turbulent PSFs. While Fig. 2 and previous research ${ }^{15-17}$ supports the premise of physical size change as a function of $r_{0}$, an examination of the PSF contours seen in Fig. 2 suggests that the overall structural characteristics of turbulent PSFs remain the same. Thus, a decrease in $r_{0}$ is equivalent to an increase in the size of the physical dimension of the turbulent PSFs. We summarize this as the following "scalability conjecture":

"PSFs from different turbulence conditions have similar morphology and primarily differ in the physical scale on which the similarities in morphology are present."

We propose using the scalability conjecture defined above to adapt our dictionary-based PSF synthesis procedure ${ }^{12}$ to varying turbulence conditions. To do this, we will assume the scalability conjecture allows PSFs with different $r_{0}$ values to be synthesized by changing the physical dimensions of PSFs synthesized at some original $r_{0}$ value. Furthermore, we assert that this conjecture also implies that a single dictionary, or a small number of dictionaries, can be used to rapidly synthesize PSFs with a broad range of $r_{0}$ values. Such an extended form of the dictionary synthesis method thereby eliminates the need to make exhaustive collections of PSFs for different conditions of turbulence. Furthermore, in Ref. 3 we disclosed that dictionary synthesis is orders of magnitude faster than conventional phase-screen and wavefront propagation-based methods. Thus, extension of the synthesis method to produce PSFs over a range of values of $r_{0}$ would make it possible to rapidly synthesize many PSFs with different turbulent characteristics. If proven to be true, this implication could dramatically increase the benefit of dictionarybased synthesis and reconstruction because continuous in situ point source data would not need to be collected to utilize sparse and redundant representations. 


\section{PSF Scaling Experiments}

\subsection{Physical Scaling of Synthesized PSFs}

As described at length in the companion article to this paper, ${ }^{3}$ a sparse and redundant representation was developed as a means to synthesize PSFs from a dictionary of PSF basis functions compiled by means of the K-SVD algorithm. ${ }^{11}$ Any PSF, which possesses the same turbulent characteristics as the PSFs used to compile the redundant representation dictionary, can be represented as a linear combination of the elements of the dictionary. The synthesis algorithm described in Ref. 3 creates the coefficients for a synthesized PSF through random perturbations to coefficients of the PSFs in the dictionary. An important constraint must be enforced to ensure that synthesis in this manner produces PSFs that are consistent with the turbulence conditions prevailing in the experimental data. The constraint is that the random perturbations must satisfy the additional condition of possessing the same summary statistics (such as mean and standard deviation) as the collection of PSFs used to compile the dictionary. This is required to ensure that synthesized PSFs are statistically representative of the turbulence process experienced by the collection of the PSFs used in the compilation of the dictionary. ${ }^{3}$

\subsection{Scaling PSFs to Extend Synthesis Capabilities}

The extension of the synthesis algorithm was achieved as a consequence of the observation stated as the scalability conjecture in Sec. 2.4. The application of the scalability conjecture to PSF synthesis is simple and direct. Given a dictionary constructed from a set of PSFs with a known value of $r_{0}$, a PSF with a different value of $r_{0}$ can be synthesized through the following process:

- First, synthesize a PSF, from the redundant representation dictionary that is at hand, by the previously reported algorithm. ${ }^{3}$

- Second, if the new PSF is to represent turbulence with a smaller value of $r_{0}$, magnify the physical scale of the newly synthesized PSF.

- Third, if the new PSF is to represent turbulence with a larger value of $r_{0}$, minify the physical scale of the newly synthesized PSF.

The rationale for the magnification and minification steps results from physical intuition of the scalability conjecture. We assume that the actual physical size of a pixel does not change in either case of magnification or minification. This is consistent with the way that different values of $r_{0}$ appear in the same focal plane, because the size of a focal plane pixel that records a PSF does not change when the $r_{0}$ value of the PSF changes. Smaller values of $r_{0}$ mean that the turbulence is more severe, which distributes the radiation from a point source over a greater region of the fixed size focal plane pixels. Thus, a synthesized PSF must be magnified in scale to behave as a PSF would in greater turbulence. A larger value of $r_{0}$, from lesser turbulence, results in the radiation from a point source being distributed in a smaller portion of the focal plane. The synthesized PSF is then minified in scale, again to exhibit behavior consistent with the turbulence conditions. In Sec. 4, we show the results of this simple procedure as validated with the experimental data from the ISO data collections.

\subsection{PSF Scaling Considerations}

Before proceeding to demonstrate specific results of employing the scalability conjecture in the synthesis of PSFs with different values of $r_{0}$, it is worthwhile to recapitulate back to the implications of the conjecture as stated previously: the scalability conjecture offers the prospect for "PSFs with different $r_{0}$ values to be synthesized by changing the physical dimensions of PSFs synthesized at some original $r_{0}$ value." Since the physical dimensions of a PSF may be changed in pursuit of the conjecture, then it is necessary to examine all aspects of the physical scale that can be present in PSF data recorded in a focal plane. Only through complete understanding of physical scale elements can collected PSF data be used to build a dictionary that is the basis for scaled synthesis. 
To begin, we note that the calculation of Eq. (1) is for only the OTF due to turbulence and does not represent other OTF characteristics of an optical system delivering PSF data to a focal plane. In fact, three different optical system characteristics need to be considered: the detector OTF, detector sampling effects on the OTF, and the OTF of the optical system delivering the PSF data into the focal plane. In Sec. 3.3.3, we also comment on the impact of speckle as it applies to our scalability conjecture.

\subsubsection{Detector and sampling OTF effects}

Sampling by a detector element integrates the flux in the area spanned by the detector. This process induces an OTF component in the spatial frequency of the recorded data. For the case where detector dimensions significantly exceed the detector spacing, which could occur by binning several individual detectors, the detector OTF can be substantial and must be corrected. Simple methods exist for correction of detector OTF in the spatial frequency domain. However, it is conventional and acceptable to ignore detector OTF correction in almost all applications where the detector dimension and detector pitch are equal, because the spatial frequency effects of the detector OTF are relatively benign in these cases. ${ }^{18}$

The spacing of the pixels in the focal plane, with respect to the highest spatial frequency supported by the limiting aperture diameter, determines if the data are undersampled, oversampled, or critically sampled with respect to the Nyquist criterion. A common measure of focal plane sampling is the optical quality factor, $Q$

$$
Q=\frac{\lambda F}{D p},
$$

where $\lambda$ is the wavelength, $F$ is the focal length, $D$ is the limiting aperture diameter, and $p$ is the pitch of the focal plane pixels. $Q=2$ is Nyquist sampling, $Q<2$ is undersampling, and $Q>2$ is oversampling. The main concern is, of course, $Q<2$, and the resultant aliasing. The optical system that acquired the ISO test data had a focal length of $0.925 \mathrm{~m}$ and detector pitch of $6.5 \times 10^{-6} \mathrm{~m}$. The laser point sources imaged had a wavelength of $0.787 \times 10^{-6} \mathrm{~m}$. Several different limiting pupils were used in the experiments, as seen previously in Table 1 . In Table 2, we summarize the $Q$ values for the four ISO test cases examined in detail in this paper and identify their corresponding sampling states. Of the four cases, Test06 is substantially oversampled, Test13 is virtually Nyquist sampled, and the two cases of Test10 and Test12 are undersampled.

The concern with undersampling is the aliasing of the spatial frequency content of the measured data in the focal plane. As it is well known, undersampling causes a "folding" of replications of the spatial frequency spectrum into spatial frequencies that are lower than the Nyquist frequency, thereby corrupting the information at folded spatial frequencies. The degree of folding determines the extent of corruption to the information content of the measured focal plane data. For certain types of focal plane content, the corruption can be substantial. For example, angled straight lines become "stair-steps," or periodic structures, such as the railroad ties that support rail tracks or parallel bars in a resolution target can exhibit a change in periodicity. However, more random structures usually do not exhibit perceptible flaws. In fact, common

Table 2 Sampling conditions for a $\lambda=0.787 \times 10^{-6} \mathrm{~m}$ source under the setup conditions described in Table 1 for the four indicated test cases.

\begin{tabular}{lcr}
\hline \hline ISO_TEST\# & $Q$ & Sampling state \\
\hline Test06 & 2.94 & Oversampled \\
Test10 & 1.76 & Undersampled \\
Test12 & 1.47 & Undersampled \\
Test13 & 1.96 & Nyquist sampled \\
\hline \hline
\end{tabular}


Hunt, Iler, and Rucci: Scalability conjecture for the Fried parameter in synthesis of turbulent...

practice is to configure focal plane detectors to purposely undersample most scenes, because the greater amplitude response in the OTF of a diffraction-limited optical system at modest folding frequencies results in more information being readily usable. ${ }^{19}$ In other words, the greatest importance in undersampling effects is the actual scene content being aliased in the folding of the spatial frequency spectrum.

While considerable literature on the effects of aliasing on scenes acquired by a diffractionlimited optical system exists, we have not found previous research on the matter of aliasing effects for turbulent PSFs recorded in a focal plane. Thus, we have no reference to serve as guidance for the cases we are examining here. It is well known that a turbulent PSF does contain frequency content to the diffraction limit of the optical aperture, which is the basis of turbulent imaging systems, such as adaptive optics. ${ }^{1}$ However, in the case of a turbulent PSF, the structure of the PSF is itself random in spatial frequency content. Therefore, the folding of undersampling causes an additional, but unknown and unknowable, randomness in the affected spatial frequencies. This has the potential to affect precision photometry measurements that depend on such spatial frequencies.

Nonetheless, it is not clear that this will cause any significant degradation of visual quality usually considered in image systems acquiring natural scenes, such as looking down on the earth from a high altitude. Indeed, because of the clear history of undersampled aliasing systems being preferred for reasons of image quality, ${ }^{19}$ we have taken the position that any undersampling of the PSFs in the ISO test data can be ignored in the following discussion and experiments. We are further encouraged in this decision by the fact that the undersampling in the two cases of Test10 and Test 12 is not severe. In Sec. 4 we do identify a situation where the undersampling may have had an impact. We intend to examine this in further detail in the future, but for the present experiments in scaling of PSFs, we have yet to find any significant deleterious effects.

\subsubsection{Optical system OTF effects}

A final, fundamental characteristic in optical system impact on measured turbulent PSF data is the OTF of the entire optical system. In the case of diffraction-limited circular aperture optics, this results in all data being passed through an OTF that creates the well-known PSF of the Airy disk. Thus, a turbulent point source observed in the focal plane of an optical system has the effect of the optical PSF convolved into the recorded PSF data. We believe the first authority to explicitly account for this in describing atmospheric turbulence was Fried, in his paper that was the original source of Eq. (1) presented above. ${ }^{20}$ If we want to synthesize a PSF that corresponds to a value of $r_{0}$ different than the $r_{0}$ value of a source PSF, we must consider whether or not the PSF to be synthesized is representative of an optical system with an identical OTF to the original $r_{0}$ measurements. If not, the PSF to be synthesized must be adjusted to represent a PSF as observed in an optical system with a different OTF.

To illustrate the point being made in the previous paragraph, consider the case of the PSF data collected in the Test06 and Test12 datasets. As recorded in Table 1, the PSFs in Test12 were measured through a limiting optical aperture twice that of Test06, and both were captured with pixels of the same physical size. If we desire to construct a PSF by physically changing the scale of Test06 PSFs to have atmospheric properties similar to Test12 PSFs then, as suggested in Sec. 3.2, we would minify a PSF synthesized from Test06 PSF data. In other words, we would distribute the energy of the PSF over a smaller region of pixels, with the size of the pixels being fixed and not changing. In general, the minification or magnification corresponding to a larger or smaller value of $r_{0}$ does not change the physical size of a pixel, but changes the distribution of PSF energy over the pixels of fixed size. Minification shrinks the distribution of energy in the focal plane, which changes the spatial frequency domain energy distribution in the Fourier domain. The Fourier uncertainty theorem tells us that a narrow space signal requires the frequency signal to be broad. ${ }^{21}$ Thus, minification spreads the spatial frequency energy over a greater range of spatial frequencies. A similar behavior applies to magnification, of course, with energy distributed in a larger area of focal plane pixels, causing a smaller distribution of spatial frequency energy in the spatial frequency domain.

Consider the wavefront that encodes a PSF as it enters the limiting aperture of the optical system. The OTF of the optical system will modify the spatial frequency content encoded in the 
wavefront. Ideally, we would like the resulting PSF in the focal plane to be unaffected by the spatial frequency response imposed by the optical system OTF. This suggests that, prior to scaling the physical dimensions of a synthesized PSF, we should correct the spatial frequency of a recorded PSF as much as possible. By doing this first, when the spatial-frequency-corrected PSF is then rescaled, we can be faithful to the optical characteristics of a different aperture by imposing an OTF that corresponds to the aperture of the target system. The net effect can be summarized as deconvolving the OTF from a PSF, scaling the PSF to a new physical size, and then convolving the scaled PSF with the OTF of the new aperture size.

The discussion in the paragraph immediately above can be summarized in the following set of steps to account for differing aperture sizes in the collection of PSF datasets and the subsequent synthesis of PSFs from the collected data:

- We assume a dataset of PSFs. These PSFs can be as originally collected by a focal plane detector or synthesized as disclosed previously. ${ }^{3}$ The PSFs possess a known value of $r_{0}$ and are collected with an aperture of size $D_{B}$, where the subscript signifies the "baseline" or starting case for the PSF data.

- We wish to scale the baseline PSFs to a new value of $r_{0}$ as collected with an optical system with an aperture that has a diameter different from that of the baseline, referred to as $D_{N}$, where the subscript signifies "new."

- The baseline aperture has a maximum (cut-off) spatial frequency of $\rho_{B}=\frac{D_{B}}{\lambda F}$, and the new aperture has maximum cut-off spatial frequency of $\rho_{N}=\frac{D_{N}}{\lambda F}$.

- We now wish to process the baseline dataset to compensate the baseline OTF. This implies multiplication by the inverse of the baseline OTF. However, as the OTF values become very small there will be substantial amplification of noise. To limit this noise amplification, we multiply the baseline OTF by the scale factor, $S_{B}$

$$
S_{B}(\rho)=\frac{2}{B(\rho)\left[1+\frac{1}{B(\rho)}\right]},
$$

where $B(\rho)$ is the OTF response for a diffraction-limited aperture of diameter $D_{B}$. The product of this fraction with the baseline OTF has the property of approximating the inverse of the OTF for values of spatial frequency remote from the cut-off, and then going smoothly to zero as the spatial frequency approaches the cut-off.

- The rescaling operation, minification or magnification, now takes place.

- After rescaling, we now multiply the rescaled data in the spatial frequency domain by the OTF of the new aperture. Thus, if the spectrum of the baseline PSF is $T_{B}$, and the OTF of the new aperture of diameter $D_{N}$ is $N(\rho)$, then the scaled and OTF corrected spectrum, $S(\rho)$, for the composite of the operations described above is

$$
S(\rho)=N(\rho) \frac{2}{B(\rho)\left[1+\frac{1}{B(\rho)}\right]} T_{B}(\rho) .
$$

It is important to note that the maximum spatial frequency that can be supported for the computation of Eq. (4) is governed by the value of the limiting spatial frequency of the two apertures.

We now present simple examples to illustrate these OTF corrections. First, we consider a case where we scale the Test06 PSFs to the conditions present in the PSFs of Test12. From Table 1, this corresponds to a baseline aperture diameter of $1.5 \mathrm{in}$. and a new aperture of 3.0 in. Figure 3 shows the various components involved in modification of the OTF properties for this scaling operation. The OTFs of Test06 and Test 12 are the dotted and dashed lines, respectively. The correction factor for Test06 data, which is $S_{B}(\rho)$ in Eq. (3), is the rising, green, dashed-dotted line, and the composite corrected OTF from Test06 aperture to Test12 aperture is the solid line lying between the OTFs of the two apertures. 


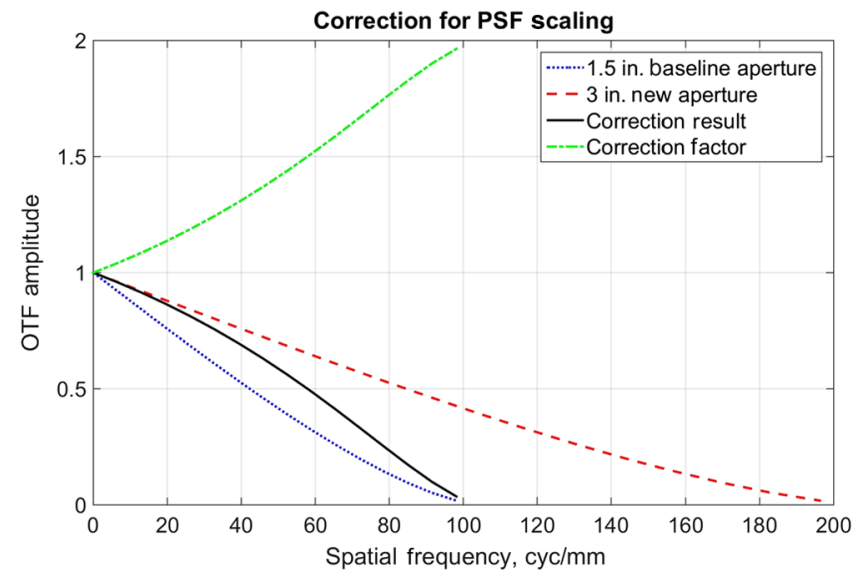

Fig. 3 Aperture OTFs and the resulting OTF correction factor for scaling Test06 PSFs to the conditions of the Test12 data.

A second case is seen in Fig. 4. Here the baseline dataset is Test10, which is to be scaled to Test13. The corresponding baseline and new apertures are 2.5 and 2.25 in., respectively. Again, the dotted and dashed lines are the baseline and new OTFs of the corresponding apertures. The correction factor $S_{B}(\rho)$ is the rising, green, dashed-dotted line and the corrected OTF lies slightly above the OTF of the new aperture. Of further interest in comparing Figs. 3 and 4 is that the correction factor applies to the baseline aperture, to correct for the OTF in the baseline.

In general, the OTF correction effect is usually a modest alteration of the spatial frequency spectrum and is seen in the cases above as slightly less than a maximum factor-of-two increase in the spatial frequencies near the cut-off of the baseline apertures. Inspection of the results of such an increase shows that little is affected in the PSFs that are modified in this way. To demonstrate the moderate nature of these OTF corrections, we present a randomly selected Test06 PSF, shown in Fig. 5(a). Figure 5(b) is the result of minification by a factor of 2 without applying any correction factor. By applying the OTF correction factor, as seen in Fig. 3, followed by minification by a factor of 2, we get the PSF in Fig. 5(c). This scaling by a factor of 2 is a "mock" scaling, as if the Test06 and Test 12 data were at $r_{0}$ values of 0.02 and $0.04 \mathrm{~m}$, respectively, rather than the actual values recorded in Table 1.

Of particular interest in Fig. 5 is the observation that the application of the correction factor before minification has little substantive impact. Visible evidence of a mild sharpening in details exists, but no significant changes of shape, structure, or contrast are present in the result, when compared to the PSF with no correction factor. This example is of particular interest, because it represents the most extreme example that we would be able to construct between different

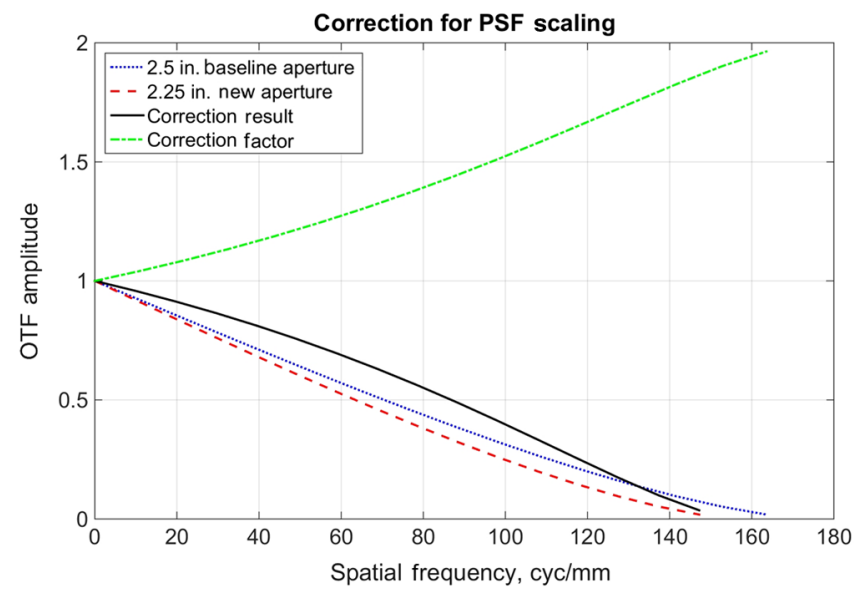

Fig. 4 Results of OTF correction for scaling Test10 PSFs to Test13 data conditions. 


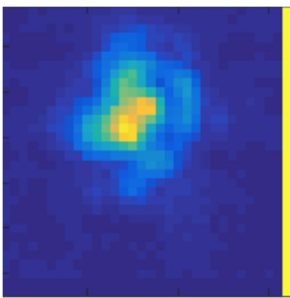

(a)

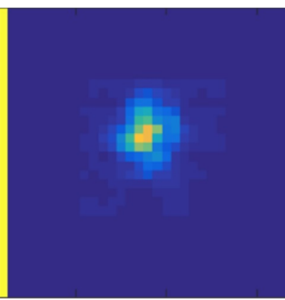

(b)

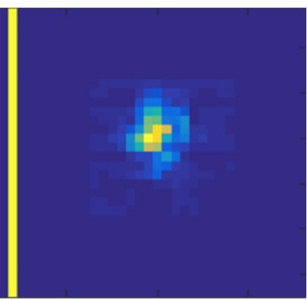

(c)

Fig. 5 A randomly selected PSF from (a) Test06, (b) minified by a factor of 2, and (c) with OTF correction prior to minification by a factor of 2 .

apertures of the ISO dataset. The conclusion is that, in many cases, we will see little substantive effect due to different aperture sizes in scaling. Indeed, if the scaling has been only as suggested from Table 1, namely the small factor associated with the actual Test06 and Test12 $r_{0}$ values of 0.03 and 0.025 , respectively, the differences would have been significantly subtler.

The correction for different aperture sizes and corresponding OTFs will be of more significance in cases where the change in scale becomes greater and the corresponding PSFs come from apertures of much greater disparity in size. Demonstrating this requires more data than were available from the ISO dataset. Regardless, the OTF correction computations, as set forth above, will be applicable in these more disparate cases.

\subsubsection{On the impact of speckle}

While instantaneous, speckled PSFs contain small speckles of the same size (i.e., $\lambda / D)$, these are only the smallest speckles expected to be found in a PSF. Many more speckles are predicted with a distribution of larger sizes. The speckle transfer function demonstrates that the most power is found in the lowest spatial frequencies, which means that larger PSF structures will dominate. These larger speckles can be envisioned as the occurrence of two or more of the smallest speckles so close together as to merge. Furthermore, $\lambda / D$ is usually interpreted as angular extent realized in an optical system, which corresponds to a physical size at the focal length where the image is formed. The physical size corresponding to the angular extent is calculated as: $s=\lambda F / D$. To retain the available information conveyed by the optical system, there must be two focal plane pixels (detectors) spaced across " $s$ " in the focal plane, placing two samples within a speckle of angular dimension $\lambda / D$. This is the Nyquist sampling rate and reaches two samples per speckle on the smallest speckles. Larger speckles may have many more samples across a speckle.

With this in mind, we direct the reader's attention to Fig. 2. These are actual PSFs recorded by the optical system discussed in Sec. 2.1. Note the absence of many distinct, isolated speckles on the order of a pixel or two in size. Instead, the PSFs manifest as mostly connected, irregular shapes spread about the focal plane. This characteristic was observed consistently in the thousands of PSFs collected in the datasets described in Table 1. Where, then, are the smaller speckles predicted by the angular extent of the optical system?

We believe the only possible answer is the discrete sampling in the focal plane at some rate related to the optimum Nyquist rate. As shown by Hunt and Breedlove in Ref. 18, a finite dimension for a detector pixel is known to act as a low-pass filter. Individual smallest speckles are of the size of this "blurring" low-pass filter. This has the effect of combining together and increasing the dimensions of the smallest speckles, diminishing the visibility of individual speckles of the smallest size, and causing some nearby speckles of smallest size to be merged into a larger structure. The additional presence of undersampling in the focal plane will make this behavior even more severe. In fact, this difference can be seen in photographs on high resolution film that, in the absence of digital sampling, clearly display individual small speckles. ${ }^{22}$

Moreover, the larger PSF structures, where more of the power in the speckle transfer function resides, will be less affected by the low-pass filtering of digital sampling in the focal plane. The result of low-pass filtering on these structures makes them even more distinct in the final PSF imagery. Therefore, in a comparison of PSFs associated with a given $r_{0}$ to PSFs associated with a much smaller value of $r_{0}$, we would expect the larger structures to be greater in extent and 
Hunt, ller, and Rucci: Scalability conjecture for the Fried parameter in synthesis of turbulent...

the presence of smaller structures to be more difficult to discern. This is what is seen in Fig. 2 and is also consistent with magnification of PSFs as proposed in our scalability conjecture.

Finally, we comment briefly on the speckle transfer function of the turbulent atmosphere in the derivation by Korff, which requires coadding the squared modulus of many Fourier transformed speckle patterns. ${ }^{23}$ This speckle transfer function is imposed on the squared modulus of the object Fourier spectrum and is inevitable in the method of Labeyrie, due to computing the sum of the squared modulus of the Fourier transformed speckle patterns. However, even after the atmospheric speckle transfer function is removed, this approach is only capable of extracting limited types of information about the object from the co-added squared modulus results, because the computed spectrum corresponds to the spatial autocorrelation of the object. To recover the actual spectrum (not the squared modulus), it is necessary to have a fool-proof algorithm for recovery of object information from the object autocorrelation. This remains a difficult problem, although some advances have occurred since the method of Labeyrie and the analysis of Korff. Since recovery of precise object information from an autocorrelation of the object remains difficult, we instead chose a metric based on the autocorrelation itself to test whether or not our method adequately addresses the speckle transfer function. This metric is discussed at length below, see Sec. 4.3.

\subsection{Initial PSF Scaling Experiments}

For our initial test of our scaling approach, we chose Test12 as the first "baseline" dataset to use for compilation of a redundant representation dictionary by the K-SVD algorithm. ${ }^{11}$ To do this, we extracted 200 PSFs from the Test12 images collected on September 22, 2015. Each PSF image consisted of a small region of $32 \times 32$ pixels from the large image frame collected by the scientific camera for the ISO test data. The 200 PSFs were selected from the $~ 3000$ available frames for being above average in the signal-to-noise ratio (SNR) of the PSF against the surrounding background. The extracted PSFs were subjected to a noise cleaning algorithm that suppressed noise in regions remote from the central core of the PSFs. ${ }^{3}$ The set of 200 PSFs from Test12 data was split into two halves. The first set of 100 PSFs was used to compile a redundant representation dictionary by the K-SVD algorithm. The remaining 100 were used as a statistical reference for testing and evaluation of the PSFs synthesized in the subsequent experiments reported in the following paragraphs.

After choosing Test12 data, and the corresponding redundant representation dictionary, as the baseline dataset for the synthesis of PSFs with new values of $r_{0}$, the cases of Test06 and Test13 were chosen for two different "new" PSF scales. The estimated $r_{0}$ value for the baseline dataset was $r_{0}=0.030 \mathrm{~m}$, as described above. Using the algorithm provided in Ref. 3, 100 new PSFs were produced by perturbations on the 100 PSFs of the Test 12 data that were reserved from the initial set of 200 PSFs. Each of these newly synthesized PSFs possessed the same physical pixel scale as the original PSFs of Test12 and were contained in $32 \times 32$ pixel frames. It was previously demonstrated that these PSFs had characteristics equivalent to the $r_{0}=0.030 \mathrm{~m}$ PSFs used to compile the dictionary. ${ }^{3}$ These synthesized PSFs were then used to compile a set of 100 PSFs for each of two new values of $r_{0}$ in the following steps:

1. The theoretical OTFs were computed from Eq. (1) for $r_{0}=0.025 \mathrm{~m}$ (Test06) and $r_{0}=$ $0.040 \mathrm{~m}$ (Test13), as shown by the dashed lines below in Fig. 6. These two $r_{0}$ values correspond to the $r_{0}$ measurements for Test06 and Test13 data, as shown in Table 1 . The inverse Fourier transform of the baseline OTF was computed to construct the corresponding PSF for $r_{0}=0.030 \mathrm{~m}$, shown as a solid blue line in Fig. 6.

2. The pixel value (measured to a fraction of a pixel) of the theoretical PSFs corresponding to the half-maximum value of the two theoretical PSFs was determined for the $r_{0}=0.025 \mathrm{~m}$ and $r_{0}=0.040 \mathrm{~m}$ cases. These pixel widths were recorded as $\rho_{25}$ and $\rho_{40}$, respectively. The value of the width at the half-maximum value for the baseline case was determined and recorded as $\rho_{B}$ using a similar procedure.

3. The Test 12 data possessed $r_{0}=0.030 \mathrm{~m}$, thus, the ratio of $\rho_{25}$ to $\rho_{B}$ produces a factor by which to magnify any PSF synthesized from the baseline dictionary to generate synthesized PSFs consistent with Test06 turbulent conditions: 


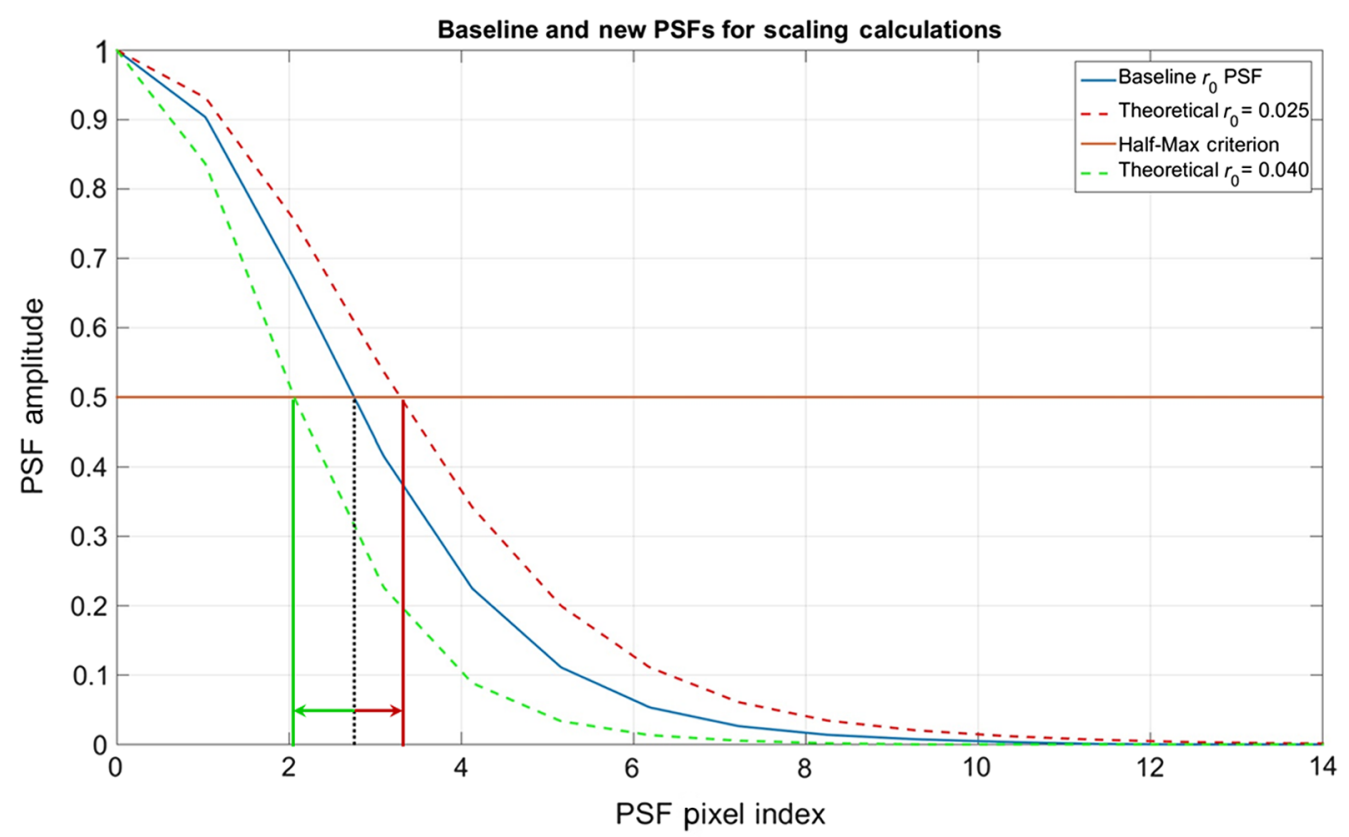

Fig. 6 The amount of magnification (indicated by the red arrow) or minification (indicated by the green arrow) used to scale our synthesized PSFs was determined through comparison of the radial width at half-maximum for the theoretical (dashed lines) and experimentally computed (solid blue line) turbulent OTFs.

Baseline magnification factor for $r_{0}=0.025 \mathrm{~m}$ is $\frac{\rho_{25}}{\rho_{B}}=1.19$.

4. The Test13 data possessed $r_{0}=0.040 \mathrm{~m}$, thus, the ratio of $\rho_{40}$ to $\rho_{B}$ produces a factor by which to minify any PSF synthesized from the baseline dictionary to generate synthesized PSFs consistent with Test13 turbulent conditions:

Baseline minification factor for $r_{0}=0.040 \mathrm{~m}$ is $\frac{\rho_{40}}{\rho_{B}}=0.68$.

5. The actual minification and magnification was carried out by the MATLAB ${ }^{\circledR}$ image processing function "imresize" as applied to the $32 \times 32$ pixel images of the synthesized PSFs. We selected the option to use bilinear interpolation for the resizing operation in the MATLAB ${ }^{\circledR}$ function.

6. In both cases, minification and magnification, the scaling changed the total volume of the PSFs. Therefore, the scaled PSFs were then renormalized to have unit total volume, as required by the nature of the OTF and image formation by PSF, i.e., to enforce conservation of energy.

Figure 6 shows this procedure for determining the scaling factors and depicts the amount of minification needed as a green arrow and the magnification needed as a red arrow.

The following images illustrate typical results produced from the scaling procedure described immediately above. Figure 7 shows a side-by-side display of a PSF synthesized from the baseline Test12 dictionary [in Fig. 7(a)] and the synthesized PSF after scaling by the magnification factor ratio $\rho_{25} / \rho_{B}$. Both PSFs show the same morphology. Figure 7(b) is slightly larger (though possibly difficult to perceive after the photoreproduction process for this publication), which is forced by the smaller value of $r_{0}$. Further, the peak of the PSF in Fig. 7(b) is clearly not as sharp as the one in Fig. 7(a), again as forced by the physical magnification of the scale.

Figure 8 shows another side-by-side display of a synthesized PSF using the baseline Test12 dictionary [in Fig. 8(a)] and the PSF after scaling by the ratio $\rho_{40} / \rho_{B}$ [in Fig. 8(b)]. Both PSFs show the same morphology. Figure $8(\mathrm{~b})$ is clearly smaller, which is forced by the larger value of $r_{0}$. Further, the peaks of the PSF in Fig. 8(b) are sharper than the ones in Fig. 8(a), again as forced by the physical minification of the scale. 


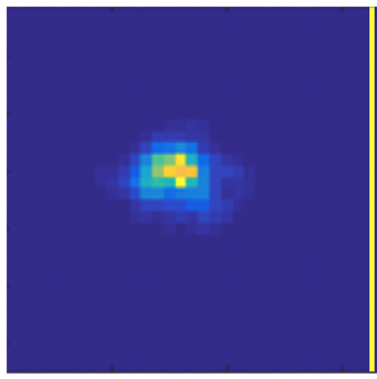

(a)



(b)

Fig. 7 Scaled PSF (magnification) from (a) $r_{0}=0.030 \mathrm{~m}$ to (b) new $r_{0}$ of $0.025 \mathrm{~m}$.

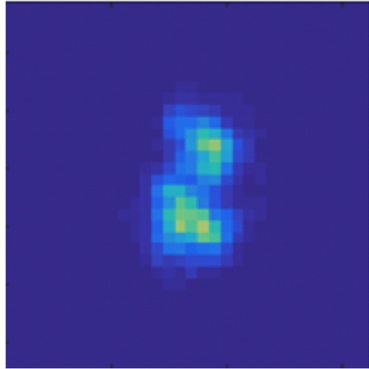

(a)

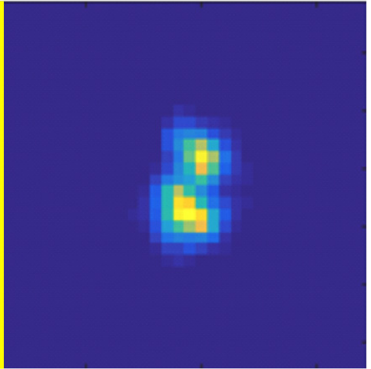

(b)

Fig. 8 Scaled PSF (minification) from (a) $r_{0}=0.030 \mathrm{~m}$ to (b) new $r_{0}$ of $0.040 \mathrm{~m}$.

A final comment is relevant to these results in the light of the discussion in Sec. 3.3 concerning the effects of different OTFs when minifying or magnifying. It is clear from the plots in Fig. 3 that the resulting correction from a factor-of-two change in the OTF cutoff spatial frequency has a moderate effect, as seen in the black line of those plots. In the cases here, with smaller scale changes that are substantially less, the corrections were much less and the decision was made to not impose them at all.

\subsection{Robustness of PSF Scaling Experiments}

To test the general tractability of our new scaling method, we also complied dictionaries of PSF basis functions from the Test10 and Test13 datasets, with derived $r_{0}=0.020 \mathrm{~m}$ and $0.040 \mathrm{~m}$, respectively. For PSFs synthesized from these dictionaries, we applied a factor-of-two scaling to minify and magnify the synthesized PSFs between $r_{0}$ of 0.020 and $0.040 \mathrm{~m}$. Figure 6 confirms the magnitude of the scale factor using the average PSF intensity profile created from the 100 reserved PSFs from Test13 and 100 PSFs synthesized from the Test10 dictionary. As indicated by the dash-dotted red line in Fig. 9, the half-maximum width of the synthesized PSF average was $\sim 10$ pixels, whereas the width of the 100 reserved Test13 PSFs was only 5 pixels. Thus, the synthesized Test10 PSFs had to be minified by a factor of 2 .

In Fig. 10, we show a similar comparison of the average PSF profile for PSFs synthesized from the Test13 dictionary and scaled to Test10 dimensions. Comparison of the PSF averages for reserved Test10 and synthesized Test10 PSFs (as seen in Fig. 9) shows that reserved Test10 PSF average is $\sim 1$ pixel larger in half-width than the corresponding average of the synthesized Test 10 PSF data. The source of this difference is currently attributed to differences in the interpolation calculations of magnification and minification. For example, minification by a factor of 2 collapses structure in two adjacent pixels into a single pixel, whereas magnification by a factor of 2 takes a single pixel and several adjacent pixels to infer two pixels. This suggests that a more sophisticated interpolation scheme (e.g., stochastic interpolation using the correlation function as the interpolation kernel) could be used to carry-out minification and magnification. Unfortunately current resources did not permit such a development. 
Hunt, Iler, and Rucci: Scalability conjecture for the Fried parameter in synthesis of turbulent...

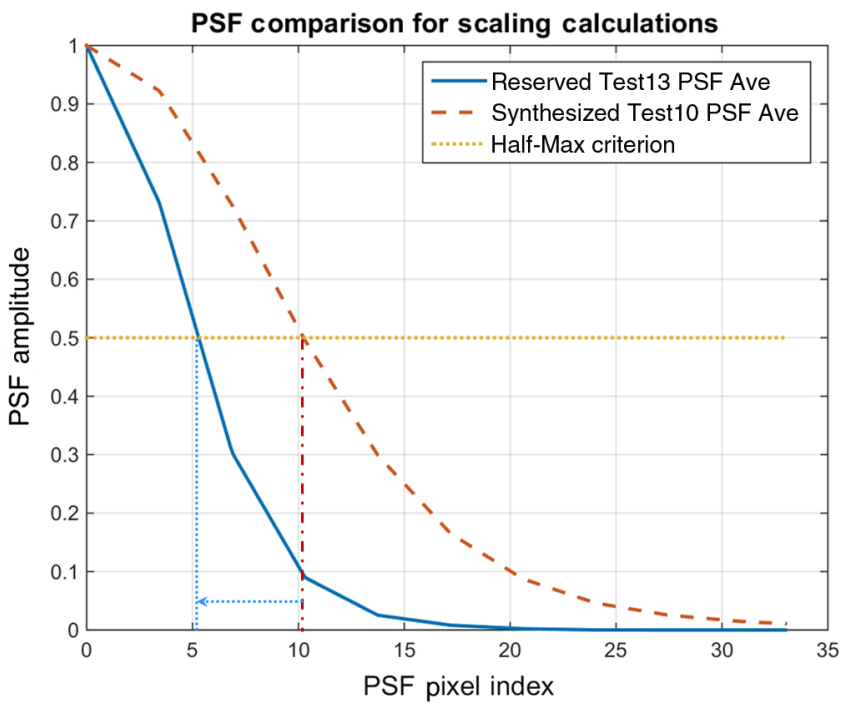

Fig. 9 PSFs synthesized from the Test10 dictionary were minified by a factor of 2 to duplicate the average OTF conditions experienced during Test13.

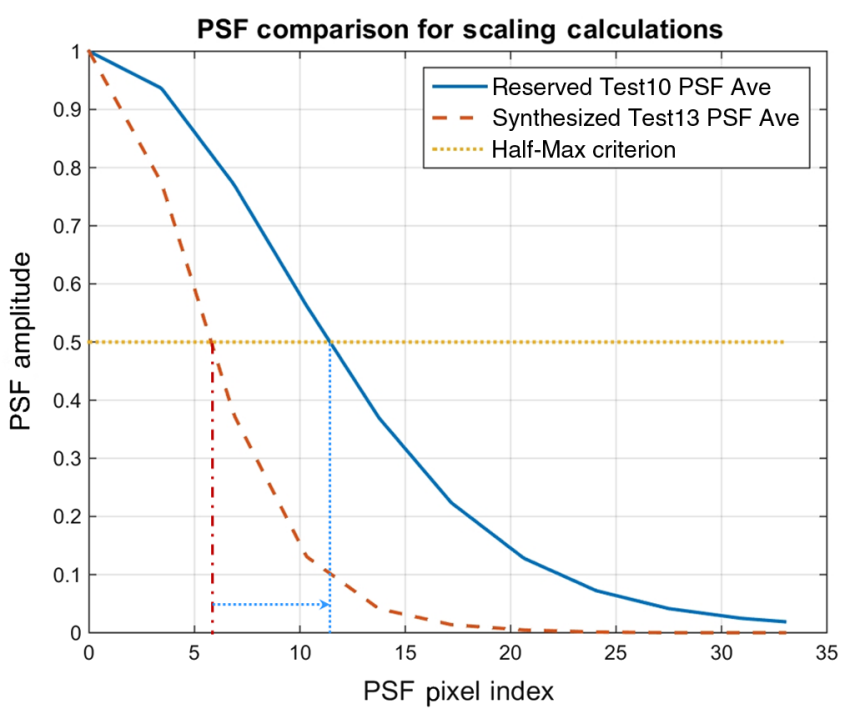

Fig. 10 PSFs synthesized from the Test13 dictionary were magnified by a factor of 2 to duplicate the average OTF conditions experienced during Test10.

Regardless of this subtle difference, the same factor-of-two scaling is revealed by comparing the average synthesized Test 13 width of slightly more than 5 pixels to the $\sim 11$ pixel width of the reserved Test10 PSFs. Figure 10 shows this scaling factor by comparison of the vertical red dashdotted and blue dotted lines. This corroborates the need to magnify synthesized Test13 PSFs by a factor of 2 to mimic the turbulence conditions of Test10. Further, the reader will see that, as measured by our statistical validation tests presented in the following section, the small differences in the PSF half-width observed do not make the corresponding scaled PSFs nonrepresentative of the targeted turbulent conditions.

\section{Results from Validation of the Scaling Algorithm}

\subsection{Validation Criteria}

In Ref. 3, we showed that the synthesis algorithm using a redundant representation dictionary produces PSFs that are equivalent to PSFs used to compile the dictionary. We wish to have the 
Hunt, Iler, and Rucci: Scalability conjecture for the Fried parameter in synthesis of turbulent...

same behavior for the PSFs that are first synthesized and then scaled, as described in the previous section above. That is, PSFs created by magnification with the ratio $\rho_{25} / \rho_{B}$ should have the same characteristics as the measured Test06 dataset PSFs, and PSFs created by minification with the ratio $\rho_{40} / \rho_{B}$ should have the same characteristics as the measured Test 13 dataset PSFs. To test this hypothesis, we subjected the scaled PSFs to the same tests as previously reported. ${ }^{3}$ For the four selected ISO data sets indicated in Table 1, we segregated the PSFs collected during the experiments into two sets: one set was used for the compilation of dictionaries and synthesis of PSFs, and one set was used as a set of PSFs that were totally divorced from dictionaries and synthesis procedures. Our comparisons then evaluated the scaled, synthesized PSFs with respect to actual, measured, PSF data that were not involved in synthesis or scaling calculations.

Five separate tests were used to evaluate the scaled PSFs:

1. The mean-square difference was computed between the average of the 100 scaled PSFs and the average of 100 PSFs actually collected in the ISO experiment. The mean-square difference was expressed as an SNR, with the average of the actual, collected PSFs being the "signal," and the mean-square difference value being the "noise."

2. We computed the mean-square difference between the average of the spatial autocorrelation functions of the 100 scaled PSFs and the average of the spatial autocorrelations of the PSFs actually collected in the ISO experiment. The results of these computations were displayed visually, as a comparison of similarity, and evaluated numerically. The mean-square difference was expressed as SNR, with the average of the autocorrelations of the actual collected PSFs being the "signal," and the difference value being the "noise."

3. The two-sided Kolmogorov-Smirnov test (KS2) was calculated between the empirical probability density function (PDF) of the intensities of the 100 scaled PSFs and the empirical PDF of the 100 PSFs actually collected in the ISO experiment.

4. The KS2 was calculated between the empirical PDFs of the central moment values of the 100 scaled PSFs and the 100 PSFs actually collected in the ISO experiment.

5. The KS2 was calculated between the empirical PDFs of the Strehl ratio values of the 100 scaled PSFs and the 100 PSFs actually collected in the ISO experiment.

The results of these five tests were all favorable with respect to demonstrating that the scaled PSFs had equivalent statistical properties to the reserved PSFs collected in the ISO experiment. The details of these tests are as follows.

\subsection{Validation Test 1}

Table 3 shows the SNR of the average difference between the PSFs synthesized from the Test12 baseline redundant representation dictionary and scaled to the $r_{0}$ values of the Test06 data, and the 100 reserved Test06 PSFs. Also shown is the SNR of the average difference between the PSFs synthesized from the Test12 baseline scaled to the Test13 $r_{0}$ values and the 100 Test13 PSFs.

The magnitude of these values indicates that the average of the synthesized PSFs possess substantial resemblance to the average of the original PSFs. Also, since the average PSF is effectively a long-time average of a point source image, this demonstrates that the scaled PSFs have long-time average behavior similar to the actual PSFs collected in the ISO experiment at the targeted $r_{0}$ values.

Table 3 SNR values between average ISO test PSFs and scaled, synthesized PSFs.

\begin{tabular}{lc}
\hline \hline Test Case & SNR of difference \\
\hline Synthesized Test12 PSFs magnified to $r_{0}=0.025 \mathrm{~m}$ & $58.6 \mathrm{~dB}$ \\
Synthesized Test12 PSFs minified to $r_{0}=0.040 \mathrm{~m}$ & $71.1 \mathrm{~dB}$ \\
\hline \hline
\end{tabular}


Table 4 SNR values between average autocorrelation of the ISO test PSFs and scaled, synthesized PSFs.

\begin{tabular}{lc}
\hline \hline Test Case & SNR of difference \\
\hline Synthesized Test12 PSFs magnified to $r_{0}=0.025 \mathrm{~m}$ & $43.7 \mathrm{~dB}$ \\
Synthesized Test12 PSFs minified to $r_{0}=0.040 \mathrm{~m}$ & $39.9 \mathrm{~dB}$ \\
\hline \hline
\end{tabular}

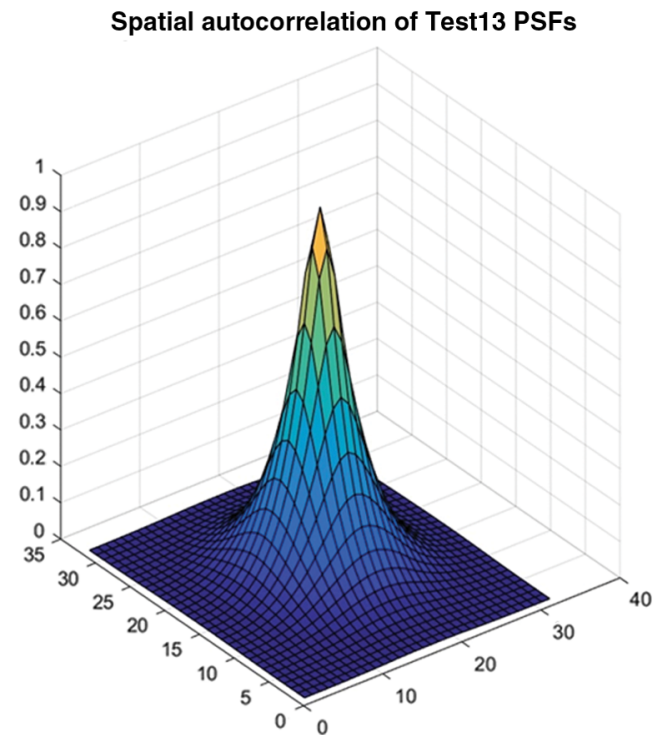

(a)

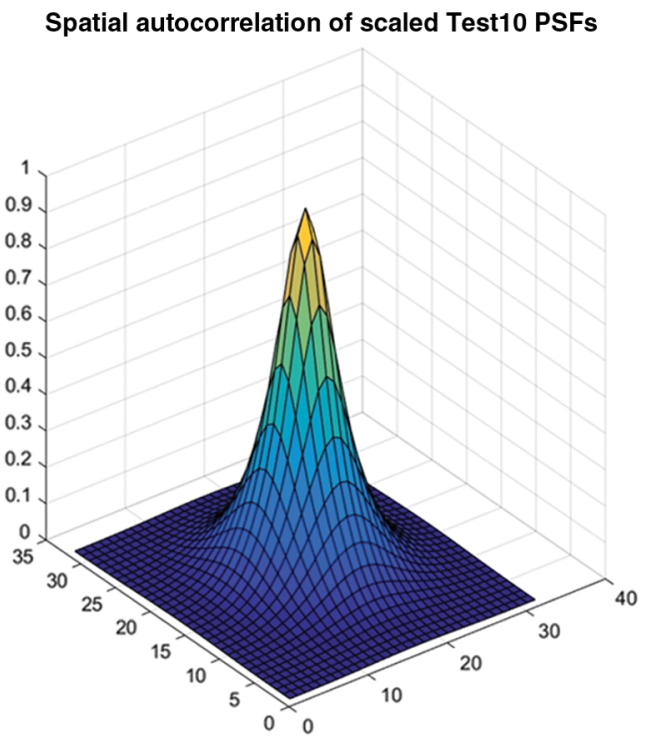

(b)

Fig. 11 Average autocorrelation of (a) the measured Test13 PSFs and (b) the scaled, synthesized PSFS.

\subsection{Validation Test 2}

Table 4 shows the SNR of the difference between the average spatial autocorrelation of the PSFs synthesized from the Test 12 baseline and scaled to the values of the Test06 data compared to the average spatial autocorrelation of the 100 reserved Test06 PSFs. Also shown is the SNR of the average spatial autocorrelation difference between the PSFs synthesized from the Test12 baseline, scaled to the $r_{0}$ values of the Test13 data, and the 100 reserved Test13 PSFs.

The autocorrelation is a measure of the similarity of a PSF at increasing distances between different locations in the PSF. The similarity in autocorrelation is also a visibly striking metric. As an example, Fig. 11 displays the average autocorrelation function of the 100 actual Test13 PSFs [in Fig. 11(a)] and the 100 PSFs synthesized to the Test13 $r_{0}$ values [in Fig. 11(b)]. The autocorrelation SNR values presented in Table 4 above quantify the degree of similarity, and the visual comparison in Fig. 11 supports the hypothesis.

\subsection{Validation Tests 3, 4, and 5}

The Kolmogorov-Smirnov (KS) test is a standard method for determining if a random variable possesses a specific distribution, e.g., distributed as a Gaussian random variable. ${ }^{21}$ In the twosample form of this test (KS2), two sets of random variables may be compared to determine if they are drawn from the same probability distribution, without specifying the actual common distribution. $^{24}$ The basic structure of the test is the computation of the empirical PDFs of the two samples, followed by evaluating a test statistic that measures the closeness of one PDF to another. The KS2 test was chosen to investigate statistical properties of the scaled PSFs with respect to the PSFs from the actual Test06 and Test13 ISO experimental collections. 
Hunt, Iler, and Rucci: Scalability conjecture for the Fried parameter in synthesis of turbulent...

Three different statistical properties of the synthesized PSFs were subjected to the KS2 test. In Test Case 3, the intensities of the synthesized and collected datasets of PSFs were compared by KS2, i.e., a test to determine if the intensity values, in the scaled PSFs and Test06 and Test13 PSFs were from the same probability distribution. In Test Case 4, the central moments of the synthesized and collected datasets of PSFs were compared by KS2, i.e., a test to determine if the distribution of intensity values about the PSF spatial centroid, in the scaled PSFs and Test06 and Test13 PSFs were from the same probability distribution. In Test Case 5, the Strehl ratio values of the synthesized and collected sets of PSFS were compared by KS2, i.e., a test to determine if an image quality metric (the Strehl ratio), for the scaled PSFs and measured Test06 and Test13 PSFs were from the same probability distribution. In all three cases, the KS2 test was applied at a significance level of 0.01 . The significance level can be interpreted as the probability of obtaining with the two samples (i.e., the 100 scaled PSFs and the 100 PSFs from the ISO Test06 and Test 13 experiments) a value of the test statistic that exceeds the actual calculated value under the null hypothesis.

In this case, the null hypothesis is: the two samples (scaled PSFs and experimentally collected PSFs) come from the same distribution. If the null hypothesis is rejected the two samples of random variables have different probability distributions. The KS2 test demonstrated, in each of the three test cases described above, that the scaled synthesized PSFs had the same properties as the Test06 and Test13 PSFs from the experimental ISO data collections. That is, the PSF intensity values, PSF central moments, and PSF Strehl ratio values each were explained by a common probability distribution for both the synthesized and measured PSFs. The actual probability distribution could be different in each of the three cases, of course.

The tests described above were the same tests as previously used to validate the original synthesis procedure disclosed in Ref. 3. Thus, the addition of the physical scaling procedure, to construct PSFs with values different than an original set of PSF sources, has been validated by the results of these same five tests.

\subsection{Robustness Tests}

In addition to the validation efforts described above using the Test06 dictionary, we also generated two dictionaries using 100 PSFs from each of the Test10 and Test13 datasets. As described previously, these dictionaries were then used to synthesize and scale additional PSFs by scaling factors that were greater than for the cases discussed immediately above. A factor of 2 distinguishes the Test 10 and Test 13 values of $r_{0}$, whereas the scaling cases discussed immediately above, from $r_{0}=0.03 \mathrm{~m}$ to $r_{0}=0.025$ and $0.04 \mathrm{~m}$, have significantly smaller scale factors. We applied the same validation criteria as described above to the factor-of-two scaled PSFs originating from these dictionaries.

For validation test 1 , we computed the average mean-square difference between the 100 Test13 synthesized PSFs scaled to $r_{0}=0.020 \mathrm{~m}$ and the 100 Test 10 synthesized PSFs scaled to $r_{0}=0.040 \mathrm{~m}$ and the average of 100 reserved Test 10 and Test 13 PSFs actually collected in the ISO experiment. The SNR values, computed the same as described in Sec. 4.1, are given in Table 5.

For validation test 2, we compared the autocorrelation of the scaled PSFs with those of the reserved Test10 and Test13 ISO datasets. Table 6 shows the SNR of the difference between the average spatial autocorrelation for the two cases we examined. Figure 12 shows three dimensional plots of the spatial autocorrelations, with the magnified Test 13 case on the top-half and minified Test 10 case on the lower-half. Note the autocorrelation appears slightly narrower and

Table 5 SNR values for the difference between the long-exposure average of PSFs synthesized for the robustness test cases and their experimentally collected counterparts.

\begin{tabular}{lc} 
Test Case & SNR of difference \\
\hline Synthesized Test13 PSFs magnified to $r_{0}=0.020 \mathrm{~m}$ & $51.3 \mathrm{~dB}$ \\
Synthesized Test10 PSFs minified to $r_{0}=0.040 \mathrm{~m}$ & $66.4 \mathrm{~dB}$ \\
\hline \hline
\end{tabular}


Table 6 SNR values between average spatial autocorrelation of the factor-of-two ISO test PSFs and their scaled, synthesized PSF counterparts.

Test Case

SNR of difference

Synthesized Test13 PSFs magnified to $r_{0}=0.020 \mathrm{~m}$

$21.1 \mathrm{~dB}$

Synthesized Test10 PSFs minified to $r_{0}=0.040 \mathrm{~m}$

$34.4 \mathrm{~dB}$

sharper in the minified Test10 case. The factor-of-two minification has the effect of collapsing PSF random structure that crosses two pixels into a single pixel, which decreases the pixel-topixel correlation. Such a decrease in pixel-to-pixel correlation makes the autocorrelation function narrower. This slight sharpening is of minimal concern, however, in view of the substantial similarity that still prevails in the comparison to the other autocorrelation function and the associated SNR value.

\section{Reserved Test10 autocorrelation}

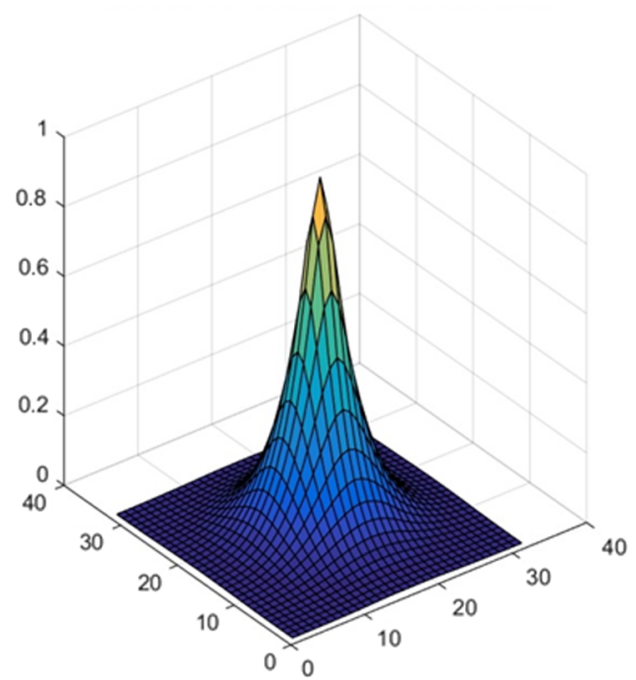

Reserved Test13 autocorrelation

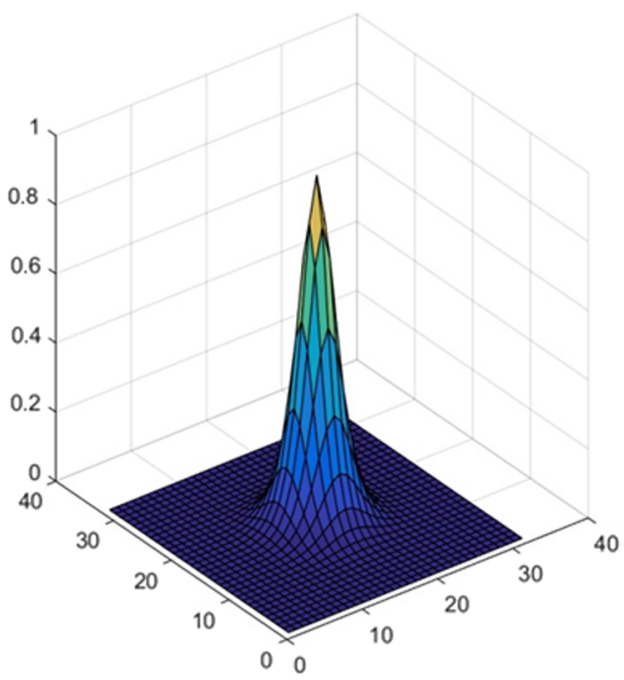

(a)

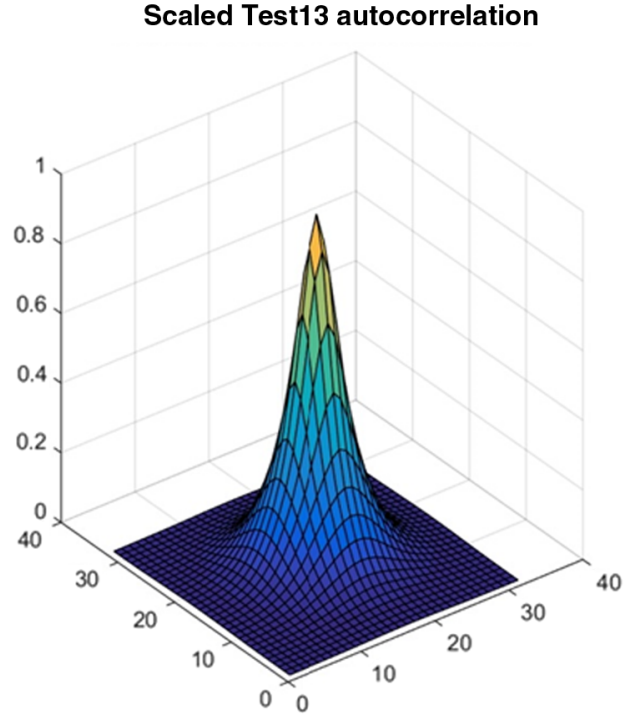

Scaled Test10 autocorrelation

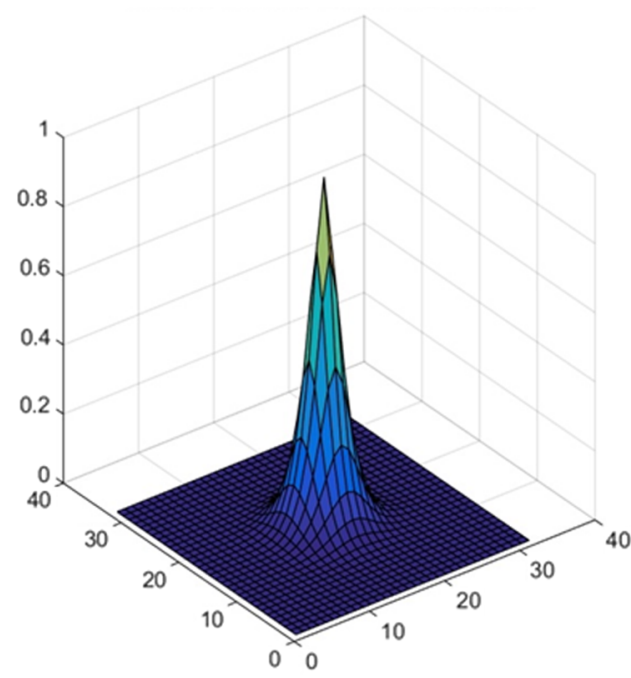

(b)

Fig. 12 Average autocorrelation for Test10 and Test13 (a) ISO PSFs and (b) scaled, synthesized PSFS. 
Finally, validation tests 3, 4, and 5 used KS2 to test the null hypothesis that the two samples (scaled PSFs and experimentally collected PSFs) came from the same distribution. The empirical probability function of their intensities, central moments, and Strehl ratios were compared in each case. For both the minified and magnified robustness tests, KS2 accepted the null hypothesis.

We wish to conclude this discussion by commenting on one aspect of the results presented in Tables 3-6. Of the four minified cases seen in these tables, three cases show SNR values significantly higher than the corresponding magnification cases. Only the case of autocorrelation SNR in Table 4 shows an SNR value for minification that is slightly less (in $\mathrm{dB}$ ) than the magnification SNR value. The specific case involves minification of Test12 data, which is the most undersampled of the four datasets, to Test13 data conditions, which is Nyquist sampled as shown in Table 2. In comparison, the minification scaling of Test10 data, which is moderately undersampled, to Test13 Nyquist data shows increased SNR in comparison to the scaling magnification of Test13 data to Test10.

These observations led us to the conclusion that the more severe undersampling errors of Test12 manifested themselves in the autocorrelation computations on the scaled PSFs. This is credible when aliasing effects are considered. Aliasing can be viewed as an increase in spatial high frequency content, created by spectral folding. Spatial high frequency content is, in general, less correlated, and that leads to the autocorrelation "width" being reduced for a smaller SNR. In any event, the SNR values are sufficiently high to be of no concern for the main point being presented seen here, i.e., the similarity of scaled PSF data to actual PSF data. The comments of Sec. 3.3, as to understanding the interactions of sampling and OTF should be recalled here, and it is our conclusion that a significantly larger amount of data needs to be collected and analyzed to understand these effects in detail.

\section{Concluding Comments and Suggestions for Further Effort}

In conclusion, we have shown a dictionary of sparse and redundant PSF representations can be used to synthesize PSFs at a different level of turbulence than the PSFs used to construct the original dictionary. This process was developed based on observations leading to the scalability conjecture: PSFs from different turbulence conditions have similar morphology and primarily differ in the physical scale on which the similarities in morphology are present. Using this conjecture, we determined the synthesis of different $r_{0}$ values can be simply achieved through geometric scaling of synthesized PSFs.

Our work discussed herein demonstrates that scaled PSFs produce PSFs consistent with measured data at the targeted $r_{0}$ values. We used several metrics to reach this conclusion: (1) the SNR difference between average ISO test PSFs and the average of our scaled PSFs; (2) the average autocorrelation of the scaled PSFs to the average autocorrelation of the PSFs actually collected during the ISO experiment; and (3) three KS2 tests to evaluate whether the intensities, central moments, and the Strehl ratios of the scaled PSFs had the same distribution as the measured ISO test PSFs. All of these tests demonstrated that the scaled, synthesized PSFs had the same properties as the PSFs from comparable experimental ISO data collections. These validations lead us to conclude that PSF synthesis by a dictionary, compiled from one set of PSF data with a fixed value of $r_{0}$, can be extended to synthesize PSFs at other values of Fried's parameter.

At this point, further experiments using collections of PSF data over wider ranges of values of $r_{0}$ are warranted, to determine how broadly the extension of Fried parameter can be applied in the manner we have described herein. As visible from Table 1, the ISO data collection was limited in the range of the Fried parameter. A broader collection of turbulence data is now necessary to verify the robustness of the scalability conjecture across a wider range of conditions. First, as discussed in Sec. 3.3, we expect OTF correction for different aperture sizes to be of more significance in cases where the change in scale becomes greater and the corresponding PSFs come from apertures of much greater disparity in size. Demonstrating this requires more data than were available from the ISO dataset. Additional data will verify the extent to which the OTF correction computations, as set forth in Eq. (4), are applicable in more disparate cases. Similarly, it could 
Hunt, Iler, and Rucci: Scalability conjecture for the Fried parameter in synthesis of turbulent...

also be used to test whether dictionary performance is improved by deconvolving the optical system OTF from measured PSFs prior to initial dictionary construction.

Finally, additional collections will also be needed to demonstrate extended capabilities for a single dictionary or to determine the number of distinct dictionaries that could be expected to encompass a greater extent of turbulence conditions. The results of such experiments, to determine the number of dictionaries that need to be compiled from actual data, will be necessary to confirm the use of a small number of dictionaries to synthesize turbulent PSFs over a wide range of $r_{0}$ values. Ideally, completion of such a collection of dictionaries would make it possible to generate turbulent atmosphere PSFs at rates that are orders of magnitude faster than the frequently used methods of Zernike polynomials and wave optics propagation. ${ }^{3}$ Development of this capability will ultimately support very efficient turbulence simulation and algorithm development over wide ranges of $r_{0}$ values.

\section{Acknowledgments}

This work was funded by the Air Force Research Laboratory, Sensors Directorate, under contract \#FA8650-12-D-1344. The authors would like to thank the AFRL EO Target Detection \& Surveillance Branch (AFRL/RYMT) for the opportunity to pursue this research and for their invaluable contributions and ideas during the planning and execution phases of this effort. This paper has been approved for public release with the PA Approval \#88ABW-2017-6386.

\section{References}

1. M. C. Roggemann and B. M. Welsh, Imaging Through Turbulence, CRC Press, Boca Raton, Florida (1996).

2. R. G. Paxman et al., "Spatial stabilization of deep-turbulence-induced anisoplanatic blur," Opt. Express 24(25), 29109-29125 (2016).

3. B. Hunt et al., "Synthesis of atmospheric turbulence point spread functions by sparse and redundant representations," Opt. Eng. 57(2), 024101 (2018).

4. R. C. Hardie et al., "Simulation of anisoplanatic imaging through optical turbulence using numerical wave propagation with new validation analysis," Opt. Eng. 56(7), 071502 (2017).

5. C. J. Carrano, "Speckle imaging over horizontal paths," Proc. SPIE 4825, 109-120 (2002).

6. R. C. Hardie et al., "Block matching and Wiener filtering approach to optical turbulence mitigation and its," Opt. Eng. 56(7), 071503 (2017).

7. S. Mallat and A. Wavelet, Tour of Signal Processing, 2nd ed., Academic Press, San Diego, California (1999).

8. R. Baraniuk, "Compressive sensing (lecture notes)," IEEE Signal Process. Mag. 24(4), 118-121 (2007).

9. J. Kovacevic and A. Chebira, "Life beyond bases: the advent of frames (part I)," IEEE Signal Process. Mag. 24(4), 86-104 (2007).

10. M. Elad, Sparse and Redundant Representations, Springer-Verlag, New York (2010).

11. M. Aharon, M. Elad, and A. Bruckstein, "K-SVD: an algorithm for designing overcomplete dictionaries for sparse representation," IEEE Trans. Signal Process. 54(11), 4311-4322 (2006).

12. J. Mairal et al., "Non-local sparse models for image restoration," in IEEE 12th Int. Conf. on Computer Vision (2009).

13. D. Zoran and Y. Weiss, "From learning models of natural image patches to whole image restoration," in IEEE Int. Conf. on Computer Vision (2011).

14. J. Tropp, "Greed is good: algorithmic results for sparse approximation," IEEE Trans. Inf. Theory 50(10), 2231-2242 (2004).

15. A. V. Sergeyev, M. C. Roggemann, and C. Demars, "Near the ground laser communication system for monitoring the statistics of turbulence: fried parameter estimation from the PSF measurements," Proc. SPIE 7685, 76850L (2010). 
Hunt, Iler, and Rucci: Scalability conjecture for the Fried parameter in synthesis of turbulent...

16. A. V. Sergeyev, M. C. Roggemann, and C. Demars, "Near the ground laser communication system: anisoplantic studies based on the PSF measurements," Proc. SPIE 8038, 80380H (2011).

17. J. P. Bos, A. V. Sergeyev, and M. C. Roggemann, "Anisoplanatism over horizontal path: comparison of theoretical and experimental results," Proc. SPIE 8246, 82460N (2012).

18. B. R. Hunt and J. R. Breedlove, "Scan and display considerations in processing images by digital computers," IEEE Trans. Comput. C-24, 848-853 (1975).

19. R. Fiete, "Image quality and [lambda]FN/p for remote sensing systems," Opt. Eng. 38(7), 1229-1240 (1999).

20. D. L. Fried, "Optical resolution through a randomly inhomogeneous medium for very long and very short exposures," J. Opt. Soc. Am. 56(10), 1372-1379 (1966).

21. A. Papoulis, The Fourier Integral and Its Applications, McGraw-Hill Publishing, New York (1962).

22. J. Dainty, "Stellar speckle interferometry," in Laser Speckle and Related Phenomena, J. C. Dainty, Ed., pp 255-280, Springer, Berlin, Heidelberg (1984).

23. D. Korff, "Analysis of a method for obtaining near-diffraction-limited information in the presence of atmospheric turbulence," J. Opt. Soc. Am. 63(8), 971-980 (1973).

24. "Kolmogorov-Smirnov test," in European Mathematical Society, 2012, Springer, https:// www.encyclopediaofmath.org/index.php/Kolmogorov-Smirnov_test (22 March 2018).

Bobby R. Hunt is a senior technical advisor with Integrity Applications Incorporated. He received his BS degree in aeronautical engineering from Wichita State University in 1964, his MS degree in electrical engineering from Oklahoma State University in 1965, and his $\mathrm{PhD}$ in systems and electrical engineering from the University of Arizona in 1967. He is the author of 180 publications in scholarly archival journals, conference proceedings, and books. His current research interests are mitigation of atmospheric turbulence, shadow imaging of space objects, and identification and classification of unresolved space objects. He is a life fellow of IEEE and a fellow of the Optical Society of America.

Amber L. Iler is a senior principal scientist at Integrity Applications Incorporated. She received her BS degree in astronomy and music from the University of Michigan in 1994 and MS degrees in physics and astronomy from Eastern Michigan University in 1996 and the University of Massachusetts in 1999, respectively. She is the author of more than 15 scientific publications and is a US patent holder. Her current research interests focus on EOIR physics, with special emphasis on exploitation of remote sensing imagery data. She is a member of SPIE.

Michael A. Rucci is a research engineer at the Air Force Research Laboratory, Wright-Patterson AFB, Ohio, USA. He received his MS and BS degrees in electrical engineering from the University of Dayton in 2014 and 2012, respectively. His current research includes day/night passive imaging, turbulence modeling and simulation, and image processing. 